\title{
Histological analysis reveals the formation of shoots rather than embryos in regenerating cultures of Eucalyptus globulus
}

\author{
Izabela Dobrowolska $^{1,2}$ - Gisele M. Andrade ${ }^{1} \cdot$ David Clapham $^{3}$. \\ Ulrika Egertsdotter ${ }^{1,4}$
}

Received: 22 April 2016 / Accepted: 10 October 2016 / Published online: 20 October 2016

(C) The Author(s) 2016. This article is published with open access at Springerlink.com

\begin{abstract}
Eucalyptus globulus is an important species in international forestry in regions with Mediterranean climates and comprises $65 \%$ of the plantation hardwood in Australia. Propagation by somatic embryogenesis would offer many advantages and its development has received much attention. Structures regenerating on explants from hypocotyls of mature zygotic embryos of E. globulus cultured on medium with NAA, reported previously to be effective for embryogenic regeneration, were analyzed morphologically and histologically to clarify their pathway of development. Analysis of series of sections revealed organogenic, rather than embryogenic, pathways of regeneration in this system. We show that protocols for propagation of E. globulus must be carefully evaluated by microscopic examination of adequate numbers of serial sections.
\end{abstract}

Keywords Eucalyptus globulus · Histological studies · In vitro culture $\cdot$ Regeneration

Izabela Dobrowolska

izabela.dobrowolska@slu.se

1 Department of Forest Genetics and Plant Physiology, Umeå Plant Science Center, Swedish University of Agricultural Sciences, 90183 Umeå, Sweden

2 Department of Cell Biology, Faculty of Biology and Environment Protection, University of Silesia, Jagiellonska 28, 40-032 Katowice, Poland

3 Department of Plant Biology and Forest Genetics, Uppsala Biocenter, Swedish University of Agricultural Sciences, 75007 Uppsala, Sweden

4 G.W. Woodruff School of Mechanical Engineering, Georgia Institute of Technology, 500 Tenth Street NW, Atlanta, GA 30332-0620, USA

\section{Introduction}

Demand for forest products is increasing globally at the same time as available forest land is decreasing. Commercial exploitation of trees necessitates improved reforestation efforts utilizing high value planting stock to increase the yields per hectare. Multiplication of elite genotypes from breeding programs and their use in plantation forestry can significantly reduce the adverse pressure on native forests and on the biodiversity of natural ecosystems (Fenning and Gershenzon 2002). Moreover, plantation forestry enables the improvement of plant materials to meet industrial requirements.

Eucalyptus species are present in over 90 countries on 22 million ha of forests, as well as 13 million ha of plantations constituting $12 \%$ of global plantations (Laclau et al. 2013). Eucalyptus is one of the most important sources of wood pulp for paper production as well as timber for construction and fuel. Moreover leaf material of eucalyptus is exploited as a source of valuable essential oils (Eldridge et al. 1994). Because of their high commercial value, Eucalyptus species are major targets for genetic improvement (de la Torre et al. 2014; Aggarwal et al. 2015), which makes the development of efficient protocols for plant regeneration urgent. While considerable genetic gains have been achieved through traditional tree breeding, we can expect additional advances exploiting biotechnological approaches such as gene characterization, in vitro culture, and genetic transformation (for references see Pinto et al. 2013). One of the most commonly worldwide cultivated Eucalyptus species is E. globulus owing to its versatility, fibre characteristics and fast growth (Patt et al. 2006).

It has been reported that in vitro regeneration of eucalyptus is possible by axillary bud proliferation (e.g. Sita and Rani 1985; Huang et al. 2014), somatic embryogenesis (SE; e.g. 
Pinto et al. 2008b; Andrade et al. 2011) and organogenesis (e.g. Hajari et al. 2006; Dibax et al. 2010). Both organogenesis and SE have been shown to be efficient techniques for the mass propagation of many species. However, in contrast to the success of micropropagation using axillary bud proliferation, effective regeneration leading to the production of vigorous plants of Eucalyptus through $\mathrm{SE}$ and organogenesis still needs to be developed. Although protocols for SE of E. globulus have been significantly improved, the reported conversion frequency of somatic embryos to plants is still relatively low (Pinto et al. 2002, 2008b; Jain 2006).

Proper classification of regenerating structures is essential for optimization of propagation procedures, and this requires histological analysis of serial sections (Yeung 1999; Haensch 2004). However, the majority of studies mainly describe protocols for plant regeneration but do not concern the histological characteristics of the process. Therefore the aim of the current study was to characterize structures from explants of E. globulus in culture conditions described previously as effective in SE regeneration (Pinto et al. 2002, 2008a, b, 2010). From histological analysis we conclude that the developing shoots are predominantly of organogenic, rather than embryogenic, origin.

\section{Materials and methods}

\section{Plant material and culture conditions}

Half-sib seeds from one mother tree were collected from an open-pollinated population of E. globulus growing in Portugal, produced from Celbi's breeding programe (Leirosa, Portugal) and kindly provided by Altri Florestal SA.

Cultures were established following the protocol described by Pinto et al. (2008a). Briefly, seeds were surface sterilized with a mixture of 1:1 absolute ethanol: hydrogen peroxide $30 \%(\mathrm{v} / \mathrm{v})$ for $15 \mathrm{~min}$. Subsequently the entire zygotic embryo was isolated from the seed by removing the seed coat under a dissecting microscope, and placed on petri dishes with induction MS medium (Murashige and Skoog 1962; Sigma-Aldrich) supplemented with $3 \mathrm{mg} / 1$ $\alpha$-naphthalene acetic acid (NAA), $30 \mathrm{~g} / 1$ sucrose, and $2.5 \mathrm{~g} / 1$ Gelrite (Haarlem, Netherlands). After 10-15 days on induction medium, germinating zygotic embryos were transferred to expression medium (the same MS medium without NAA) to induce SE. After 3-5 weeks, when globular structures originating from the hypocotyl regions were observed, these hypocotyl regions were isolated, transferred to fresh expression medium in petri dishes and subcultured onto fresh medium every 4 weeks until histological analysis. During all steps of culture, explants were kept in darkness at $24^{\circ} \mathrm{C}$; to keep conditions as constant as possible, explants were evaluated for not more than 3-5 min every $2-3$ weeks under a Zeiss stereomicroscope (Semi 2000-C; Zeiss, Germany) equipped with a white light source and Canon digital camera (PowerShot G-9; Canon Inc, Japan).

\section{Histological characterization of regenerating structures}

For histological investigations, the samples included regenerating structures together with the part of the explant from which they originated. Samples were fixed overnight at room temperature in 3\% glutaraldehyde (Sigma-Aldrich) in $0.2 \mathrm{M}$ phosphate buffer, $\mathrm{pH}$ 6.8. Samples were washed three times in phosphate buffer, dehydrated gradually in an ethanol series, transferred through propylene oxide and embedded in Epon812 resin (Sigma-Aldrich) as described (Dobrowolska et al. 2001). Serial Sections (3 $\mu \mathrm{m}$ thick; longitudinal or transverse) were cut using Hydrax M40 microtome (Zeiss, Germany) and attached to microscope slides with Haupt's adhesive. Sections were stained with PAS-reaction + toluidine blue (Sigma-Aldrich; Barlow and Kurczynska 2007) and with Sudan IV to localize lipid substances (Sigma-Aldrich; O'Brien and McCully 1981) and analyzed under a Nikon light microscope equipped with colour camera (Eclipse Ni; Nikon Instruments Inc., The Netherlands). Six regenerating structures from each of six explants (a total of 36 samples) were analyzed.

\section{Results}

\section{Morphological observations of explants with regenerating structures}

All explants were brown and fragile on the expression medium, with a rough irregular surface. Each explant had formed dozens of green or yellowish regenerating structures. It was possible to distinguish several types of regenerating structures: shoots at different stages of development (Fig. 1a, b), nodular structures (Fig. 1c), and structures with dome-like shape or with mild dorsiventral symmetry (Fig. 1d). Nodular structures and very young shoots (twoleaf stage) were semi-transparent and yellowish or light greenish. Shoots more advanced in development and domelike structures were green; structures with dorsiventral symmetry were also green or sometimes brown (Fig. 1e). All regenerating structures were easy to detach from the explants. In some cases examination of separated structures under the microscope revealed remnants of explant tissue still attached to the nodular structure.

\section{Histology of regenerating structures}

Since the purpose of our studies was to confirm the embryogenic origin of structures regenerating in the previously 


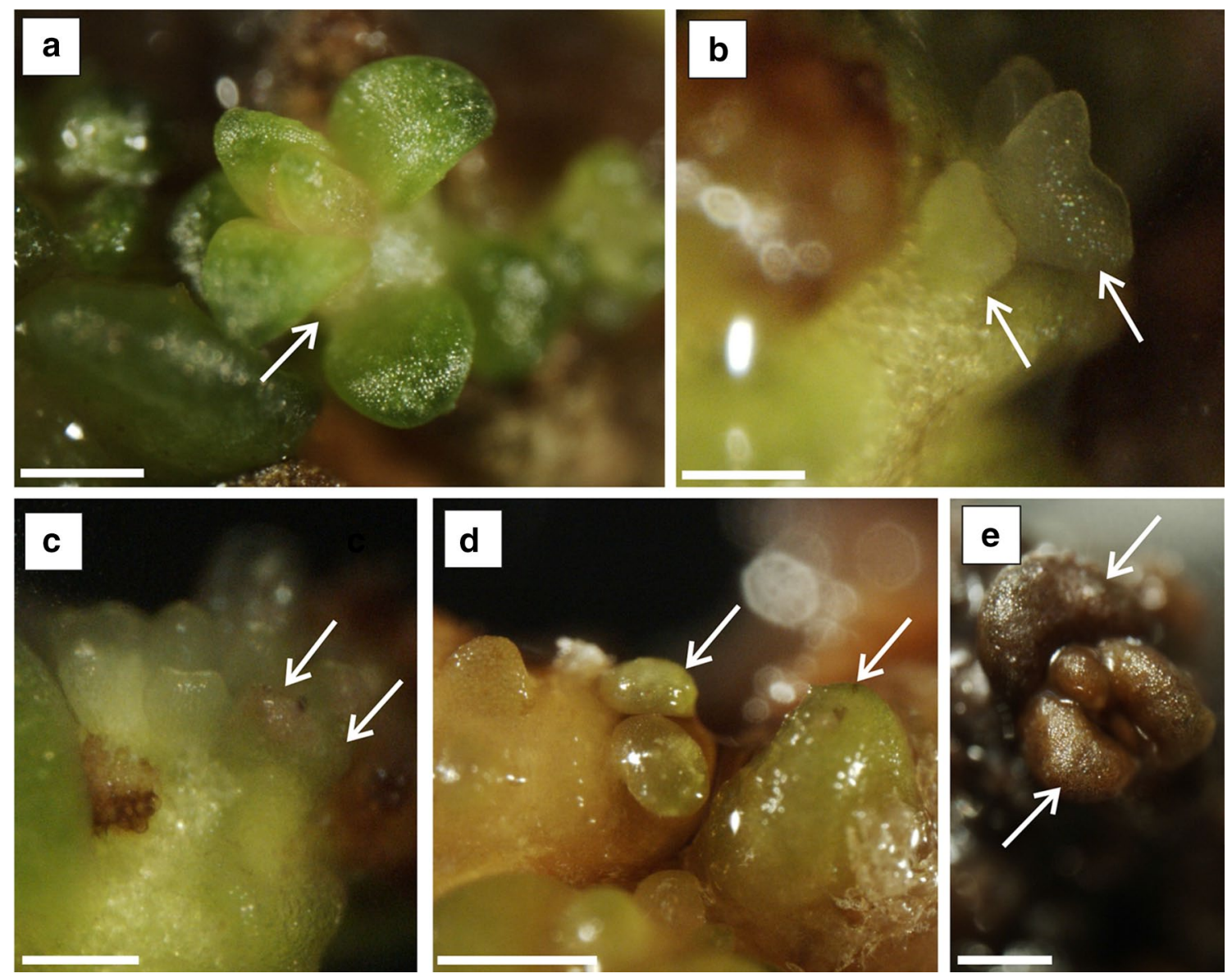

Fig. 1 Morphology of structures regenerating from explant from mature E. globulus zygotic embryo. a Arrow indicates shoot with leaves. bArrows indicate shoots in two-leaf stage of development. c Arrows indicate nodular structures. d Arrows indicate green dome-like shaped regenerating structures. e Arrows indicate brown dome-like shaped regenerating structures. Scale bar a, d $800 \mu \mathrm{m} ; \mathbf{b}$ $100 \mu \mathrm{m}$; c $200 \mu \mathrm{m}$; e $500 \mu \mathrm{m}$. (Color figure online)

layer of vacuolated cells with visible cuticle and in some of them periclinal division walls were present (Fig. 2a, b). In some areas they were separated from the explant by a layer of cells continuous with the layer of cells with cuticle (Fig. 2a). Both parenchymatous and surface cells were rich in polyphenol deposits (Fig. 2a-c).

The series of transverse sections of nodular structures revealed that they grew out of a subepidermal cortex of the explant and the globular shape did not continue below the surface of the explant (Fig. 2d). Nor were they continuously separated from the explant by a distinct layer of cells (Fig. 2e, f) with a lipid layer at the side of the explant (Fig. 2g). The cells separating the nodular structures from the explant were strongly vacuolated and rich in polyphenol deposits, and in some of them division walls were present (Fig. 2f). Furthermore, groups of tracheary elements were visible (Fig. 2d, e). They were in continuation with tracheary elements arranged in a helix or circle in the explant part beneath the nodular structure (Fig. 2h, i). 

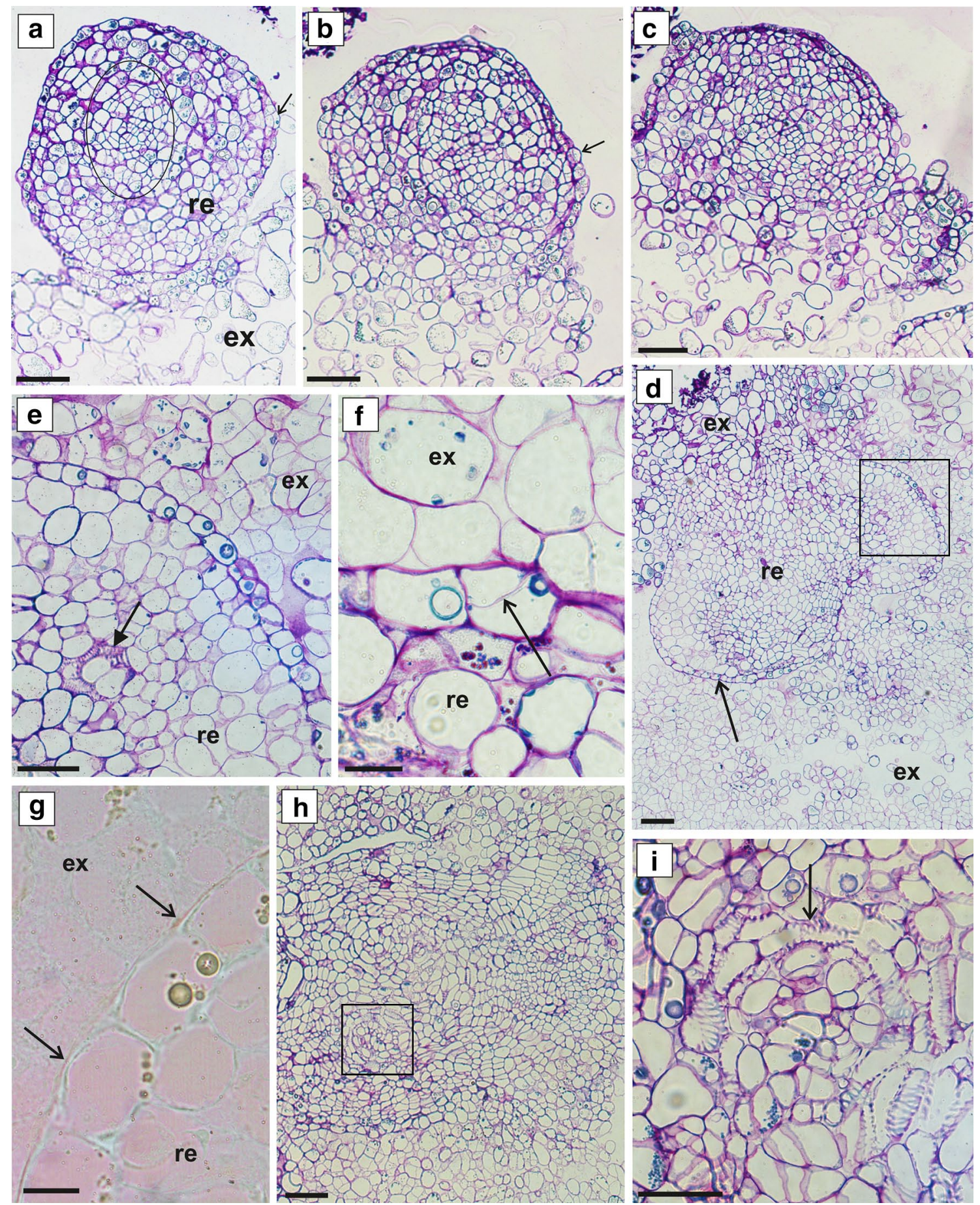

Fig. 2 Histology of nodular structures regenerating from explant from mature E. globulus zygotic embryo. Bright field; a-f, h, i PAS + toluidine blue staining (blue deposits in cells-polyphenols); g Sudan IV staining (black arrows indicate cuticle); re regenerating structure, ex explant. a-c Nodular structure growing above connection zone between regenerating structure and explant, series of longitudinal sections $(\mathbf{a}, \mathbf{b} \approx 45 \mu \mathrm{m} ; \mathbf{b}, \mathbf{c} \approx 54 \mu \mathrm{m})$, black arrow indicates divided epidermis cell, black circle outlines area with intensively dividing cells. $\mathbf{d}-\mathbf{i}$ Nodular structure growing both above and below the connection zone- transverse sections. $\mathbf{d}-\mathbf{g}$ Section below connection zone between regenerating structure and explant, black-edge square on "d" outlines fragment shown on "e", black arrow indicates layer of cells separating regenerating structure from explant, black arrow with filled arrowhead - tracheary element. $\mathbf{h}-\mathbf{i}$ Section of explant just beneath nodular structure, black-edge square on "h" outlines fragment shown on "i", black arrow indicates tracheary element. Scale bara-c, d, h $100 \mu \mathrm{m}$; e, i $50 \mu \mathrm{m} ; \mathbf{f}, \mathbf{g} 20 \mu \mathrm{m}$. (Color figure online) 


\section{Two-leaf-stage shoot}

The morphological characteristics of structures classified by us as young shoots may suggest either organogenic or embryogenic origin. However, the analysis of a series of longitudinal sections did not reveal root poles in these structures, only leaves and shoot apical meristems being visible (Fig. $3 \mathrm{a}-\mathrm{c}$ ). In the transition zone between a young shoot and the explant, parenchyma cells belonging to the shoot and explant differed from each other and it was possible to distinguish explant tissue from shoot tissue. Both shoot and explant parenchyma cells in this area were strongly vacuolated and isodiametric but shoot cells were smaller than explant cells (Fig. 3a, b, d). Moreover, explant parenchyma cells seemed to be richer in polyphenol deposits (Fig. 3a, $\mathrm{b}, \mathrm{d})$. In the transition zone in the shoot part we observed tracheary elements (Fig. 3b, e). The shoots were covered by an epidermis. The vacuoles of the epidermal cells were rich in polyphenol deposits (Fig. 3a-e), and in the vicinity of the epidermis, oil glands were visible (Fig. 3a, c). No starch grains were observed.

\section{Discussion}

Media supplemented with NAA were reported to be effective in inducing production of somatic embryos in cultures of various Eucalyptus species such as E. citriodora (Muralidharan and Mascarenhas 1987), E. tereticornis (Prakash and Gurumurthi 2005) or E. camaldulensis (Prakash and Gurumurthi 2010). Direct SE was reported in explants from hypocotyls obtained from germinated seeds (Prakash and Gurumurthi 2010), but the most frequently described indirect $\mathrm{SE}$ is from explants from mature and immature zygotic
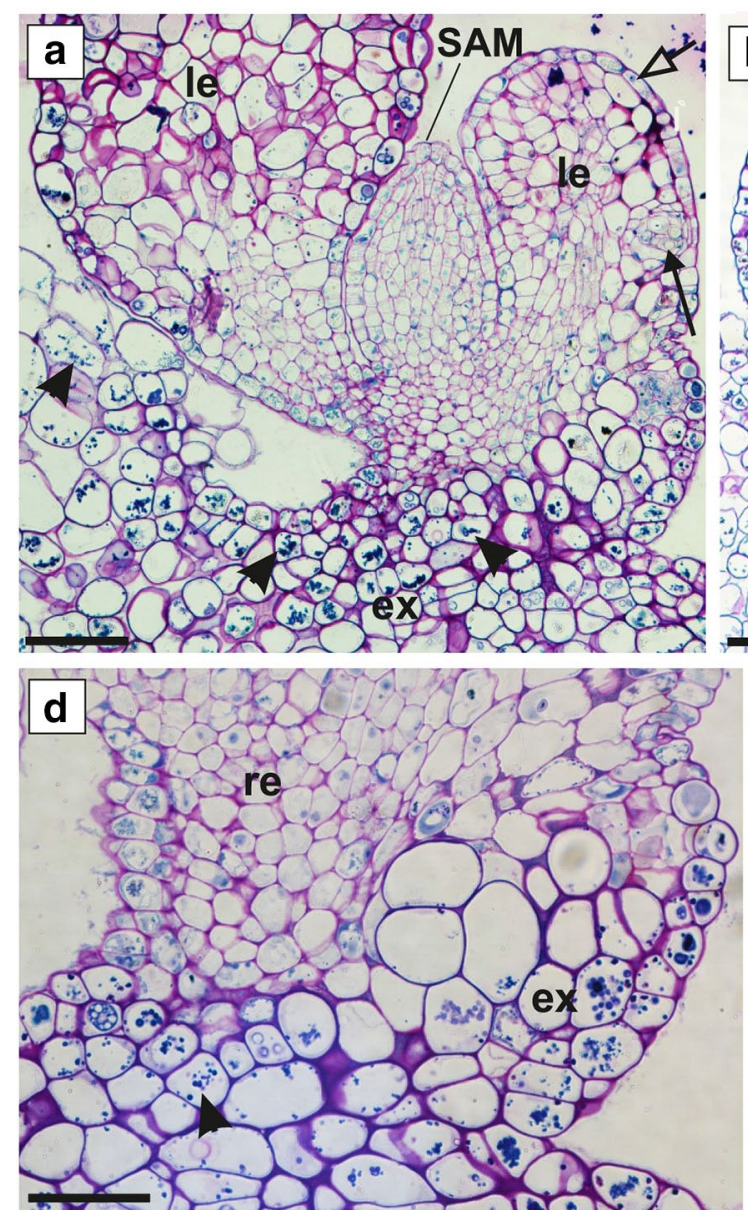

Fig. 3 Histology of two-leaf-stage shoot regenerating from explant from mature E. globulus zygotic embryo. Bright field; PAS + toluidine blue staining. a-c Series of longitudinal sections through the regenerating structure $(\mathbf{a}, \mathbf{b} \approx 90 \mu \mathrm{m} ; \mathbf{b}, \mathbf{c} \approx 144 \mu \mathrm{m}) ; \mathbf{d}$ transition zone between explant and regenerating structure; e fragment of connection zone between explant and regenerating structure outlined by
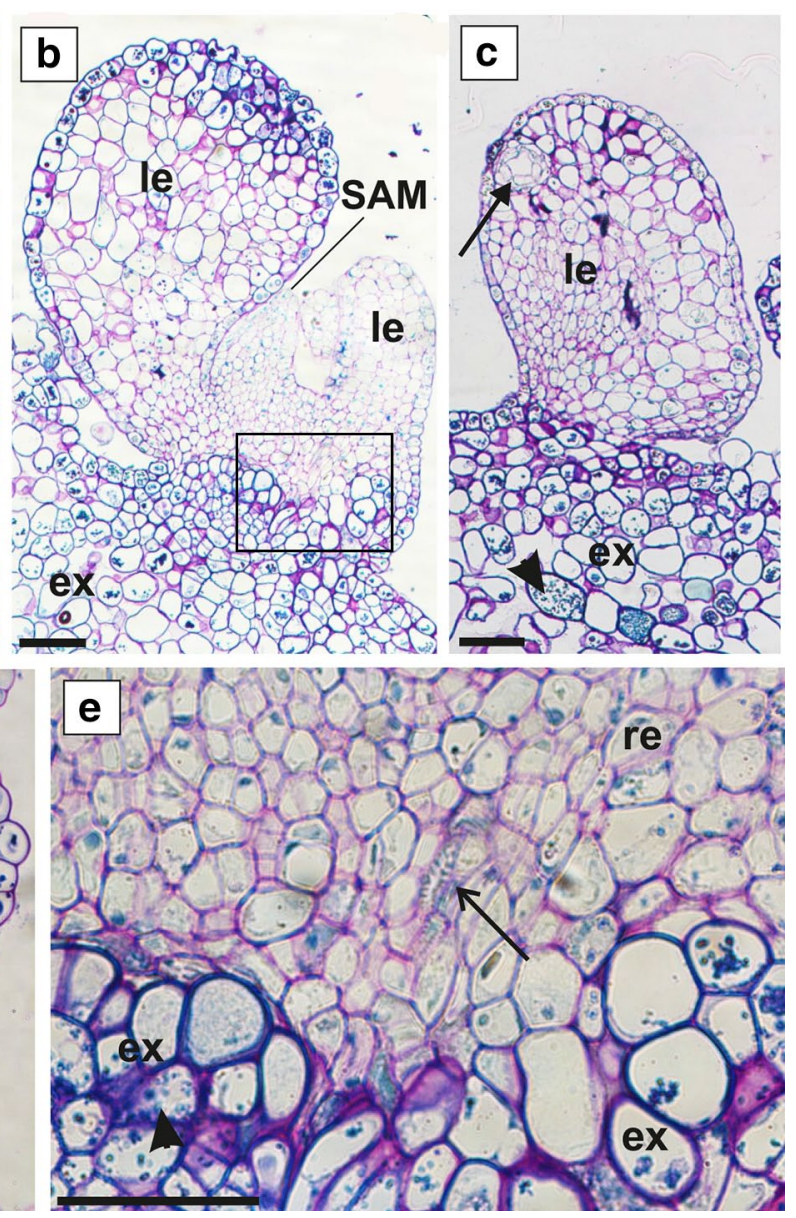

black-edged square on "b"; SAM shoot apical meristem, le leaf, ex explant, re regenerating structure, black arrow with empty arrowhead indicates epidermis, black arrows with filled arrowhead - oil gland, black arrows with opened arrowhead - tracheary elements, black arrowheads - polyphenol deposits. Scale bar a-c $100 \mu \mathrm{m} ; \mathbf{d}-\mathbf{e} 50 \mu \mathrm{m}$ 
embryos (Nugent et al. 2001; Pinto et al. 2002, 2008a, b; Prakash and Gurumurthi 2005), and young (Pinto et al. 2002) and mature plants (Corredoira et al. 2015). Most publications on SE in Eucalyptus concern attempts to develop a reliable protocol for induction of SE (Nugent et al. 2001; Prakash and Gurumurthi 2005, 2010; Pinto et al. 2008b; Corredoira et al. 2015). Only a few reports concern histological and ultrastructural (Pinto et al. 2002), and genetic characteristics of somatic embryos (Pinto et al. 2004). E. globulus is regarded as extremely recalcitrant to regeneration through SE. Some authors observed the formation of somatic embryos on different kinds of explant of E. globulus but the embryos exhibited structural abnormalities and their conversion to plants was not effective (Bandyopadhyay et al. 1999; Nugent et al. 2001). Pinto et al. (2002) for the first time described a reproducible protocol for SE in $E$. globulus from mature embryos. Histological analysis is a powerful tool when developing new methods for organogenesis or SE procedures (Yeung 1999). Since the biological specimens are three-dimensional objects, conclusions based on two-dimensional sections, without taking into consideration the third dimension, can easily lead to mistaken conclusions (Yeung 1999). Histological analysis of series of sections avoids interpretation errors and is especially important for understanding new culture systems (Yeung 1999). For example, SE in Pelargonium hypocotyl cultures was reported in many papers over the years (see references in Haensch 2004), but histological examinations were not conducted. Detailed histological analysis made later on by Haensch (2004) revealed that structures classified previously as somatic embryos did not possess features typical for somatic embryos. These findings led to a more critical view over regeneration protocols for Pelargonium. Since $E$. globulus is of great industrial importance, improvement of micropropagation technique based on SE with all its advantages is of special interest. However, the reported conversion frequency of somatic embryos to plants is still relatively low (Pinto et al. 2002, 2008b) and the lack of proper identification of structures regenerating in vitro significantly impedes optimization of culture conditions.

Some histological analysis of structures regenerating after using a protocol described as effective for SE in $E$. globulus was conducted (Pinto et al. 2008a). However the cross sections of clusters of somatic embryos presented by Pinto et al. (2008a) do not demonstrate the independence of the explant tissue from the presumed somatic embryos. A typical histological feature of somatic embryos is the presence of a root pole. Moreover, it has been reported that starch is present at the radical poles of somatic embryos, as is the case with zygotic embryos (Kurczynska et al. 2007, 2012). Our histological analysis, like that of Pinto et al. (2008a), revealed that the regenerating structures resembling putative somatic embryos at the cotyledonary stage were devoid of root poles and starch grains in the basal region. Additionally, vascular tissue was present in the distal region of these structures, near to the explant. The presence of a vascular tissue connection between the regenerating structure and the explant is the other feature distinguishing organogenesis from embryogenesis (Wicart et al. 1984; Haensch 2004). We did not observe vascular connection between regenerating structures and explant. Nevertheless the lack of other features typical for embryos, such as bipolarity and distribution of starch grains at the radical pole, suggests the non-embryogenic origin of these structures and their classification as young shoots.

We also analyzed series of sections for nodular structures resembling globular embryos. Globular somatic embryos have been previously reported to consist of small cells with dense cytoplasm and large nuclei as were observed in species that include Daucus carota (Schiavone and Cooke 1985) and Nigella sativa (Banerjee and Gupta 1976). Both in the nodular structures that we obtained and in the structures described by Pinto et al. (2008a, 2010) as globular somatic embryos, the majority of the cells were parenchymatous and vacuolated. Our nodular structures that emerged from explants were anchored in subepidermal cortex. Detailed histological analysis of series of transverse sections revealed that in more proximal regions, below the explant surface, the shape of these structures was irregular. Moreover, sections of the subepidermal parts of the explants from which these structures emerged showed that they were separated from the explant tissue in some areas by deposition of lipid substances on the periclinal cell walls. It was described earlier that buds regenerating from hypocotyls of E. globulus remained in a subepidermal position in the early stages; subsequently the shoots elongated and initiated new leaves while the epidermis became disrupted (Azmi et al. 1997). Also during the regeneration of E. camaldulensis from cotyledonary explants, caulinar meristems developed in subepidermal positions (Dibax et al. 2010). As a result of subsequent intensive cell divisions meristematic agglomerates broke the explant epidermis and emerged above the surface of the explant (Dibax et al. 2010). Therefore the nodular structures we observed seem to correspond with an early stage of bud formation. It has also been reported that in cultures of woody plant species differentiating from dedifferentiated cells, structures called nodules are commonly seen. Nodules have been described as a developmental pathway parallel to embryogenesis and have been shown to display a high capacity for plant regeneration via organogenesis (McCown et al. 1988). The formation of nodules has previously been reported in cultures of various Eucalyptus species (Ito et al. 1996). Trindade and Pais (2003) have described that under specific conditions nodules can be formed on explants from seeds, cotyledons, hypocotyls and juvenile leaves of E. globulus 
(Trindade and Pais 2003). Nodules were proposed to be anatomically characterized as independent cohesive cell masses with defined protoderm, which do not fall apart even with rather strong physical disruption. However, it has later been reported that both in E. globulus (Trindade and Pais 2003) and in other species such as Linum (Salaj et al. 2005), nodules were easily separated from the explant. Our nodular structures and other regenerating structures could also easily be detached from explants, probably owing to the fragility of explant tissue. Another typical feature is vascularization (McCown et al. 1988) which we observed in the nodular structures. Nevertheless no sign of internal vascularization has previously been observed in E. globulus (Trindade and Pais 2003).

\section{Conclusions}

Our results indicate that protocols for the in vitro propagation of Eucalyptus and other species must be carefully evaluated by the microscopic examination of adequate numbers of serial sections. Histological studies with serial sectioning of the material at different stages of culture allow a three-dimensional reconstruction of the material and provide credible information about the origin of regenerating structures and their identity, which is essential in studies of tissue culture systems. Although we cannot completely exclude the formation of somatic embryos using the protocol presented here, our studies clearly show that histological analysis is a basis for the improvement of current protocols for the micropropagation of E. globulus, whether through somatic embryogenesis or adventitious shoot formation.

Acknowledgments We thank Altri Florestal SA (Portugal) for E. globulus Labill. seeds. We also thank Ewa U. Kurczynska from Department of Cell Biology at University of Silesia (Poland) for valuable comments.

Author contributions Study conception and design-UE, GMA, ID; preparing of plant material-GMA; sectioning of samples and microscopy analysis - ID; interpretation of data-ID; drafting of manuscript-ID; critical revision-DC, UE; final approval of the version to be published-UE, GMA, DC, ID.

\section{Compliance with ethical standards}

Conflict of interest The authors declare that they have no conflict of interest.

Open Access This article is distributed under the terms of the Creative Commons Attribution 4.0 International License (http://creativecommons.org/licenses/by/4.0/), which permits unrestricted use, distribution, and reproduction in any medium, provided you give appropriate credit to the original author(s) and the source, provide a link to the Creative Commons license, and indicate if changes were made.

\section{References}

Aggarwal D, Kumar A, Reddy MS (2015) Genetic transformation of endo-1,4- $\beta$-glucanase (Korrigan) for cellulose enhancement in Eucalyptus tereticornis. Plant Cell Tiss Organ Cult 122:363-371

Andrade G, Shah R, Johansson S, Pinto G, Egertsdotter U (2011) Somatic embryogenesis is a tool for forest tree improvement: a case-study in Eucalyptus globulus. BMC Proc 5(Suppl 7):P128

Azmi A, Noin M, Landré P, Prouteau M, Boudet AM, Chriqui D (1997) High frequency plant regeneration from Eucalyptus globulus Labill. Hypocotyls: ontogenesis and ploidy level of the regenerants. Plant Cell Tiss Organ Cult 51:9-16

Bandyopadhyay S, Cane K, Rasmussen G, Hamill JD (1999) Efficient plant regeneration from seedling explants of two commercially important temperate eucalypt species-Eucalyptus nitens and E. globulus. Plant Sci 140:189-198

Banerjee S, Gupta S (1976) Embryogenesis and differentiation in Nigella sativa leaf callus in vitro. Physiol Plant 38:115-120

Barlow P, Kurczynska EU (2007) The anatomy of the chi-chi of Ginkgo biloba suggests a mode of elongation growth that is an alternative to growth driven by an apical meristem. J Plant Res 120:269-280

Corredoira E, Ballester A, Ibarra M, Vieitez AM (2015) Induction of somatic embryogenesis in explants of shoots cultures established from adult Eucalyptus globulus and E. saligna $\times E$. maidenii trees. Tree Physiol. doi:10.1093/treephys/tpv028

de la Torre F, Rodríguez R, Jorge G, Villar B, Álvarez-Otero R, Grima-Pettenati J, Gallego PP (2014) Genetic transformation of Eucalyptus globulus using the vascular-specific EgCCR as an alternative to the constitutive CaMV35S promoter. Plant Cell Tiss Organ Cult 117:77-84

Dibax R, Quisen RC, Bona C, Quoirin M (2010) Plant regeneration from cotyledonary explants of Eucalyptus camaldulensis Dehn and histological study of organogenesis in vitro. Braz Arch Biol Technol 53:311-318

Dobrowolska I, Kurczynska EU, Dmuchowski W (2001) Anatomy of abscission zone of Betula pendula (Roth.) leaves from trees growing under different levels of pollution. Dendrobiology 46:13-19

Eldridge K, Davison J, Harwood C, Van WG (1994) Eucalypt domestication and breeding. Clarendon Press, Oxford

Fenning TM, Gershenzon J (2002) Where will the wood come from? Plantation forests and the role of biotechnology. Trends Biotechnol 20:291-296

Haensch K-T (2004) Morpho-histological study of somatic embryolike structures in hypocotyl cultures of Pelargonium $\times$ hortorum Bailey. Plant Cell Rep 22:376-381

Hajari E, Watt MP, Mycock DJ, McAlister B (2006) Plant regeneration from induced callus of improved Eucalyptus clones. S Afr J Bot 72:195-201

Huang Z, Ouyang L, Li Z, Zeng F (2014) A urea-type cytokinin, 2-ClPBU, stimulates adventitious bud formation of Eucalyptus urophylla by repressing transcription of rbohl gene. Plant Cell Tiss Organ Cult 119:359-368

Ito K, Doi K, Tatemichi Y, Shibata M (1996) Plant regeneration of eucalypts from rotating nodule cultures. Plant Cell Rep 16:42-45

Jain (2006) An update on overall recent progress on somatic embryogenesis in forest trees. In: Suzuki K, Ishii K, Sakurai S, Sasaki S (eds) Plantation technology in tropical forest science. Springer, Tokyo, pp 113-122

Kurczynska EU, Gaj MD, Ujczak A, Mazur E (2007) Histological analysis of direct somatic embryogenesis in Arabidopsis thaliana (L.) Heynh. Planta 226:619-628

Kurczynska EU, Potocka I, Dobrowolska I, Kulinska-Lukaszek K, Sala K, Wrobel J (2012) Cellular markers for somatic embryogenesis. In: Sato K-I (ed) Embryogenesis. InTech, Rijeka, pp 307-332 
Laclau J-P, de Moraes Gonçandrade JL, Stape JL (2013) Perspectives for the management of eucalypt plantations under biotic and abiotic stresses. For Ecol Manage 301:1-5

McCown BH, Zeldin EL, Pinkalla HA, Dedoph RR (1988) Nodule culture: a developmental pathway with high potential for regeneration, automated micropropagation, and plant metabolite production from woody plants. In: Hanover JW, Keathley DE (eds) Genetic manipulation of woody plants. Plenum, New York, pp 149-166

Muralidharan EM, Mascarenhas AF (1987) In vitro plantlet formation by organogenesis in E. camaldulensis and by somatic embryogenesis in Eucalyptus citriodora. Plant Cell Rep 6:256-259

Murashige T, Skoog F (1962) A revised medium for rapid growth and bioassays with tobacco tissue cultures. Physiol Plant 15:473-497

Nugent G, Chandler SF, Whiteman P, Stevenson TW (2001) Somatic embryogenesis in Eucalyptus globulus. Plant Cell Tiss Org Cult 67:85-88

O'Brien TP, McCully ME (1981) The study of plant structure. Principles and selected methods. Termarcarphi Pty. Ltd., Melbourne

Patt R, Kordsachia O, Fehr J (2006) European hardwoods versus Eucalyptus globulus as a raw material for pulping. Wood Sci Technol 40:39-48

Pinto G, Santos C, Neves L, Araújo C (2002) Somatic embryogenesis and plant regeneration in Eucalyptus globulus Labill. Plant Cell Rep 21:208-213

Pinto G, Loureiro J, Lopes T, Santos C (2004) Analysis of the genetic stability of Eucalyptus globulus Labill. somatic embryos by flow cytometry. Theor Appl Genet 109:580-587

Pinto G, Park Y-S, Silva S, Neves L, Araújo C, Santos C (2008a) Factors affecting maintenance, proliferation, and germination of secondary somatic embryos of Eucalyptus globulus Labill. Plant Cell Tiss Organ Cult 95:69-78

Pinto G, Silva S, Park Y-S, Neves L, Araújo C, Santos C (2008b) Factors influencing somatic embryogenesis induction in Eucalyptus globulus Labill.: basal medium and anti-browning agents. Plant Cell Tiss Organ Cult 95:79-88

Pinto G, Silva S, Neves L, Araújo C, Santos C (2010) Histological changes and reserve accumulation during somatic embryogenesis in Eucalyptus globulus. Trees 24:763-769

Pinto G, Araújo C, Conceição S, Neves L (2013). Plant regeneration by somatic embryogenesis in Eucalyptus spp.: current status and future perspectives. South For 75:59-69

Prakash MG, Gurumurthi K (2005) Somatic embryogenesis and plant regeneration in Eucalyptus tereticornis Sm. Curr Sci 88:1311-1316

Prakash MG, Gurumurthi K (2010) Effects of type of explant and age, plant growth regulators and medium strength on somatic embryogenesis and plant regeneration in Eucalyptus camaldulensis. Plant Cell Tiss Organ Cult 100:13-20

Salaj J, Petrovská B, Obert B, Pret'ová A (2005) Histological study of embryo-like structures initiated from hypocotyl segments of flax (Linum usitatissimum L.) Plant Cell Rep 24:590-595

Schiavone FM, Cooke TJ (1985) A geometric analysis of somatic embryo formation in carrot cell cultures. Can J Bot 63:1573-1578

Sita JL, Rani BS (1985) In vitro propagation of Eucalyptus grandis L. by tissue culture. Plant Cell Rep 4:63-65

Trindade H, Pais MS (2003) Meristematic nodule culture: a new pathway for in vitro propagation of Eucalyptus globulus. Trees 17:308-315

Wicart G, Mouras A, Lutz A (1984) Histological study of organogenesis and embryogenesis in Cyclamen persicum Mill. tissue cultures: evidence for a single organogenetic pattern. Protoplasma 119:159-167

Yeung EC (1999) The use of histology in the study of plant tissue culture systems - some practical comments. In Vitro Cell Dev Biol Plant 35:137-143 